\title{
Coherent combining of second harmonic generators by active phase control of the fundamental waves
}

\author{
Alice Odier $^{1}$, Anne DuréCu ${ }^{1}$, JeAn-Michel Melkonian ${ }^{1}$, LAURent Lombard ${ }^{1}$, \\ Michel Lefebvre ${ }^{1}$, Pierre Bourdon ${ }^{1, *}$ \\ ${ }^{1}$ ONERA - The French Aerospace Lab, BP 80100, 91123 Palaiseau cedex, France \\ *Corresponding author: alice.odier@onera.fr
}

Received XX Month XXXX; revised XX Month, XXXX; accepted XX Month XXXX; posted XX Month XXXX (Doc. ID XXXXX); published XX Month XXXX

\begin{abstract}
Coherent beam combining (CBC) by active phase control could be useful for power scaling fiber-laser-pumped optical frequency converters. However, a fast phase modulator operating at the frequency-converted wavelength, a non-standard component, would be necessary. Fortunately, nonlinear conversion processes rely on a phase-matching condition allowing for indirect phase control using standard phase modulators. In this paper, coherent combining of second harmonic generators is demonstrated in both birefringent and quasi phase matching schemes in $\mathrm{CW}$ regime. Phase control operates at the fundamental wavelength, using all-fibered electro-optic modulators. An excellent beam combination is achieved with a residual phase error of $\lambda / 30$ on the second harmonic wave. (C) 2017 Optical Society of America
\end{abstract}

OCIS codes: (140.3515) Lasers, frequency doubled; (140.3298) Lasers, beam combining; (190.5040) Phase conjugation.

http://dx.doi.org/10.1364/OL.99.099999

Coherent beam combining (CBC) with active phase control is a very convenient technique to power scale laser sources far beyond the limitations of a single amplifying chain.

Through CBC, the power from multiple laser sources can be summed up, giving access to an overall power beyond thermal effects and optical damage threshold limitations for solid state lasers [1,2], or nonlinear effects limitations for fiber lasers [3-6].

On top of giving access to unrivalled levels of laser power, CBC with active phase control also offers great versatility. In a tiledaperture configuration, this technique is capable of performing beam shaping, wavefront correction for propagation through atmospheric turbulences and beam steering [7-9]

Nowadays, lasers are commonly used with nonlinear optical components, in order to extend their operating wavelength range or to provide wavelength tunability through parametric frequency conversion. Pumped by a near infrared laser, second and third harmonic generators (SHG and THG) give access to the visible and ultraviolet part of the optical spectrum [10,11]. Conversely, downfrequency conversion of a near infrared laser can generate longer wavelengths, up to the mid-infrared bands of transparency of the atmosphere (3-5 $\mu \mathrm{m}$ and $8-12 \mu \mathrm{m})$ [12-14].

However, these frequency converters are not immune to maximum power limitations, as the nonlinear crystals used in these devices often suffer from low damage thresholds [15]. These damage threshold limitations are even more critical as frequency converters' efficient operation relies on the use of high peak power levels, potentially increased by thermal focusing effects [16].

Thus, nonlinear devices could also benefit from CBC with active phase control techniques, for power scaling above their standard limitations. Nonetheless, performing CBC by use of frequency converters requires the capability to control and to drive the phase of the converted waves. Unfortunately, phase modulators (or phase actuators) operating at specific wavelengths emitted by the nonlinear devices are not always available or offer limited performances. This issue becomes more stringent for tunable devices.

To our knowledge, the use of $\mathrm{CBC}$ in conjunction with frequency conversion has been scarcely reported. The developers of laserguide-star systems have used CBC to increase the power of a 1178$\mathrm{nm}$ fiber laser, then doubling this high power laser in a single nonlinear crystal to produce the targeted 589-nm wavelength [17]. A similar configuration has been demonstrated at different wavelengths for atom interferometry application [18]. Frequency doubling has also been used to build a 6 to 1 coupler in a single nonlinear crystal: a non collinear phase matching was implemented to combine 6 fundamental waves and phase control them to generate a single output second harmonic beam $[19,20]$. However, in these configurations, the use of a single nonlinear crystal for frequency conversion limits the total power in these experiments, due to the damage threshold of the crystal.

In this paper, we experimentally demonstrate that $\mathrm{CBC}$ with active phase control can be implemented for second harmonic generators, without controlling the phase of the second harmonic wave. We explain how CBC can operate with sole control of the fundamental wave phase, and result nevertheless in efficient coherent combining of the harmonic waves. 
This original CBC configuration can lead to very convenient and simple ways to combine and power scale frequency converters, as it rely on the same components and electronics as CBC of telecom lasers (for instance the fundamental wave lasers).

The physical principle takes full advantages of the phase relationship existing between the coupled waves in any nonlinear process. First, we introduce basic equations to express the electric fields for the fundamental wave and the second harmonic wave. For the sake of simplicity without loss of generality, we assume the beams to be both linearly polarized, and use scalar quantities. We limit our analysis to the case of continuous wave with monochromatic emission. The fundamental and harmonic electric fields can be written as:

$E_{\omega}(z, t)=a_{\omega}(z) \exp \left(i\left(k_{\omega} z-\omega t+\varphi_{\omega}\right)\right)+c . c$.

$E_{2 \omega}(z, t)=a_{2 \omega}(z) \exp \left(i\left(k_{2 \omega} z-2 \omega t+\varphi_{2 \omega}\right)\right)+c . c$.

(2)

Where $\omega$ and $2 \omega$ are the frequency of the fundamental and the harmonic wave and $k_{\omega}$ and $k_{2 \omega}$ their wave vectors. $a_{\omega}(z)$ and $a_{2 \omega}(z)$ are assumed to be real numbers, the phase of the complex amplitudes of the electric fields being $\varphi_{\omega}$ and $\varphi_{2 \omega}$.

In a lossless $\chi^{(2)}$-active nonlinear crystal, the evolution of the fundamental and second harmonic beams is driven by the coupled equations:

$$
\begin{aligned}
& \frac{d a_{\omega}(z)}{d z} \exp \left(i \varphi_{\omega}\right)= \\
& 2 i \kappa_{\omega} a_{2 \omega}(z) a_{\omega}(z) \exp (i \Delta k z) \exp \left(i\left(\varphi_{2 \omega}-\varphi_{\omega}\right)\right) \\
& \frac{d a_{2 \omega}(z)}{d z} \exp \left(i \varphi_{2 \omega}\right)= \\
& \left.2 i \kappa_{2 \omega} a_{\omega}(z)^{2} \exp (-i \Delta k z) \exp \left(2 i \varphi_{\omega}\right)\right)
\end{aligned}
$$

with $\Delta k=k_{2 \omega}-2 k_{\omega}$.

$\kappa_{\omega}$ and $\kappa_{2 \omega}$ are complex numbers that depend on the nonlinear susceptibility of the frequency converter medium, and on its refractive indices for the fundamental and harmonic waves. Solving these coupled equations under the assumption that the fundamental wave is not depleted (i.e. for low level of power), one can show that the energy is efficiently transferred from the fundamental wave to the harmonic wave if the phase matching condition is fulfilled, ie $\Delta k=0$. It also results in a phase relation: $\varphi_{2 \omega}=\varphi_{0}+2 \varphi_{\omega}$ where $\varphi_{0}$ stands for the phase component of the $i \kappa_{2 \omega}$ complex number [21].

Similarly, in the quasi phase matching (QPM) case, high conversion efficiency can be achieved in a periodically poled crystal when the relation between phases of the fundamental and second harmonic wave is $\varphi_{2 \omega}=2 \varphi_{\omega}$ [21].

In the case of critical phase matching, where walk-off is present, an offset of phase is added during the propagation, and there's still a fixed phase relation between the fundamental and harmonic waves.

For higher power levels, the depletion of the fundamental wave is significant and the propagation equations are solved using Jacobi elliptic functions [22]. Nevertheless, the phase relations remain valid in the undeleted regime in both the perfect and the quasi phase matching cases.

Due to those phase relations between the interacting fields, driving the fundamental wave phase leads to a full control of the second harmonic wave phase. For instance, in the QPM case, a $\varphi_{1}$ phase shift induced on the fundamental wave will result in a $2 \varphi_{1}$ second harmonic phase variation.

Two experimental set-ups were built to demonstrate such indirect phase control and to validate the theoretical principle presented before: the first setup is a QPM scheme using two periodically poled lithium niobate (PPLN) crystals pumped by $1.55-\mu \mathrm{m}$ Er:fiber lasers and the second setup is a standard phase matching scheme using two birefringent lithium borate (LBO) crystals pumped by $1.064-\mu \mathrm{m}$ Yb:fiber lasers. Given the wavelength of fundamental waves, standard high bandwidth allfiber electro-optic phase modulators operating at the fundamental wavelength can be used.

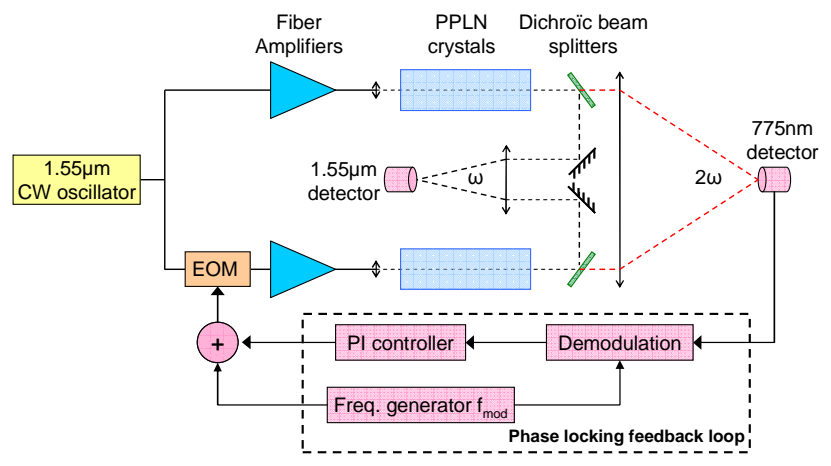

Fig. 1. Schematic of the experimental set-up for demonstrating $\mathrm{CBC}$ of two SHG PPLN modules by phase-control of the fundamental wave. A similar setup is used for the SHG in LBO crystals.

Figure 1 describes the PPLN experiment. Two 1.55- $\mu \mathrm{m}$ Er:fiber amplifiers are seeded by a master oscillator, in this case a 10-mW laser diode (JDS uniphase) operating at $1553 \mathrm{~nm}$. On both optical channels, power amplification is achieved using commercial erbium-doped fiber amplifiers $(2 \mathrm{~W}$ polarization maintaining Keopsys amplifiers). One of the $1.55-\mu \mathrm{m}$ fiber amplifiers is phasecontrolled using a high bandwidth (400 MHz) fiber-coupled electro-optic modulator (EOM). This EOM (Photline) provides both the low amplitude phase modulation for frequency-tagging and the proper phase shifts to compensate for phase fluctuations. We use two different PPLN crystals in this experiment (5-cm long and 4-cm long respectively). The crystal temperatures are separately tuned to maximize SHG efficiency at $1553 \mathrm{~nm}$. Fundamental and harmonic waves are filtered out using dichroic beam splitters. The two fundamental beams are overlapped to interfere on an InGaAs fast photodetector (Thorlabs DET 10C) while the two harmonic beams are overlapped to interfere on a silicon fast photodetector (Thorlabs DET 10A). A lock-in amplifier (Stanford Research SR 830) delivers the frequency-tagging modulation at $56.6 \mathrm{kHz}$ on the EOM. It also demodulates the interference signal from the 775-nm sensitive photodetector, to extract an error signal. This error signal is locked-in to 0 by a proportional integral (PI) controller used to drive the EOM induced phase shift.

The layout for the LBO SHG experiment is similar. The master oscillator is a RIO Orion laser module at $1064 \mathrm{~nm}$. Both $1.06 \mu \mathrm{m}$ power amplifiers are homemade ytterbium-doped fiber amplifiers, each of them capable of delivering up to $30 \mathrm{~W}$ power. A fibercoupled electro-optic modulator at $1.06 \mu \mathrm{m}$ controls the phase of one of the optical channels. Both LBO crystals are identical (4-cm long LBO manufactured by Cristal Laser and heated-up at $150^{\circ} \mathrm{C}$ ). 
In this experiment, the lock-in detection is performed by MiniCircuits operating in the MHz range. The tagging frequency is fixed at $10 \mathrm{MHz}$. Two silicon photodetectors are used in this experiment, the first one on the 1.06- $\mu \mathrm{m}$ interference pattern and the second one on the 532-nm interference pattern. SHG CBC is achieved using the 532-nm interference signal for phase control.

To measure absolute phase values, we implemented I/Q detection in both experiments and for both the fundamental and second harmonic beams.

The goal of these preliminary experiments is to demonstrate the feasibility of indirect phase control, not to establish records in terms of SHG efficiency. Thus, the SHG efficiency is quite low, around a few \% only $1.4 \%$ for LBO experiment and $2 \%$ for PPLN experiment) as the lasers are continuous-wave operating. SHG efficiency maximization would have required some enhancement: higher pump power, longer crystal or cavity enhanced SHG, a far more complex experiment than needed here.

Before performing indirect phase control we need to verify the phase relation between the fundamental and the second harmonic waves.

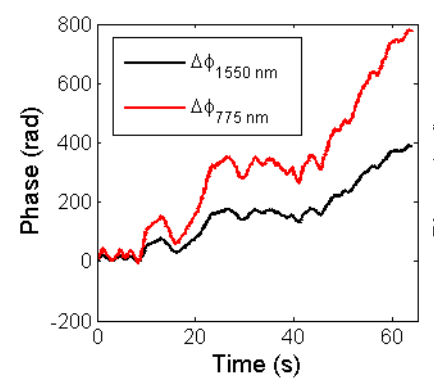

(a)

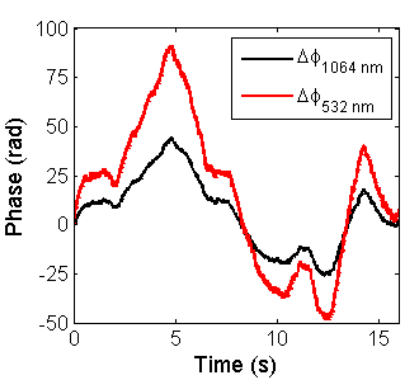

(b)
Fig. 2. Phase measurements when the control loop is open: (a) for PPLN experiment, (b) for LBO experiment.

Figure 2 presents an example of free phase fluctuation measurements (i.e. when the phase-locking control loop is inactive) for fundamental and harmonic wave (a) in the PPLN experiment (b) in the LBO experiment. We are able to verify directly that the second harmonic phase follows the theoretically expected twice the fundamental phase value. Therefore, indirect phase control can be used to perform coherent beam combining by active phase control.

Figure 3a presents the intensity measured in the center of the interference patterns by each photodetector, in the PPLN $1.55-\mu \mathrm{m}$ SHG combining experiment. As long as the phase-control loop is open, the interference signal fluctuates freely between destructive and constructive states. When the feedback-loop is closed, the 775nm interference signal is locked on a constructive interference and the signal measured by the photodiode is kept near its maximum value. The time-averaged combining efficiency is excellent: the residual phase error is $\lambda / 30 \mathrm{rms}$ for the second harmonic wave (assessed from direct phase measurements using I/Q detection). Same result has been achieved with the LBO combining experiment.

To explore further the residual phase fluctuations, we calculated the phase fluctuation spectral density (PSD) of the second harmonic wave. As expected, comparison of the PSD measurements when the phase control loop is open and closed (see figure $3 \mathrm{~b}$ ) indicates a significant damping of these phase fluctuations when phase-locking is active.

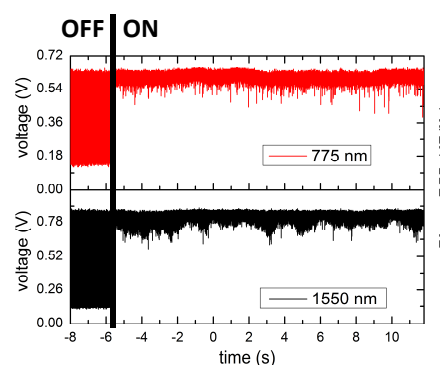

(a)

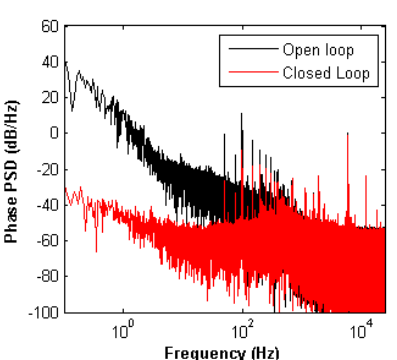

(b)
Fig. 3. Results for the PPLN experiment: (a) Time evolution of the interference signals of the fundamental (black) and the harmonic beams (red) when the phase-control feedback loop is off (loop is open) and on (loop closed). (b) Phase spectral density of the second harmonic wave when the phase control loop is open or closed

When the control loop is open, the main phase fluctuations are low frequency (i.e. below the kilohertz range). Above a few $\mathrm{kHz}$, the PSD curves overlap, which is consistent with the 4-kHz cut-off frequency of the feedback loop we measured through impulse response experiments. The response time of the active control loop was assessed adding a sudden stair-step phase shift command on the EOM, and measuring the recovery time needed for the harmonic waves interference signal to return to the constructive state of interference.

In the PPLN experiment, when the control loop operates on the interference signal between the second harmonic beams, the fundamental beams interference locks on an arbitrary state, nor systematically constructive nor destructive. Manually adding proper optical path offset allows driving the interference signal to the constructive state, as presented in figure 3a. However, phase locking is slightly less efficient for the fundamental waves than for the harmonic waves: the residual phase error on the fundamental beams was measured to be $\lambda / 20 \mathrm{rms}$ which still corresponds to a good combining efficiency. It shows that efficient coherent combining is achievable on both fundamental and harmonic waves simultaneously in this very favourable case. This efficiency difference is due to the contribution of experimental sources of phase fluctuations like optical path disturbances or electronic noise on the fundamental waves residual phase difference.

In the LBO experiments, we observed some strong and fast varying additional phase fluctuations, leading to the impossibility to achieve simultaneous fundamental and harmonic phase locking. To better understand the origins of those additional phase fluctuations, we needed to study absolute phase values.

The phase spectral densities of the fundamental and the second harmonic waves when the control loop is closed are presented in figure $4 \mathrm{a}$ and figure $4 \mathrm{~b}$ for the PPLN and LBO experiments.

Due to the additional sources of phase fluctuations on the fundamental waves, the integral of the PSD with respect to the frequency is higher for the fundamental waves. It corresponds to stronger fundamental phase fluctuations when the phase control loop operates on the second harmonic interference signal. 


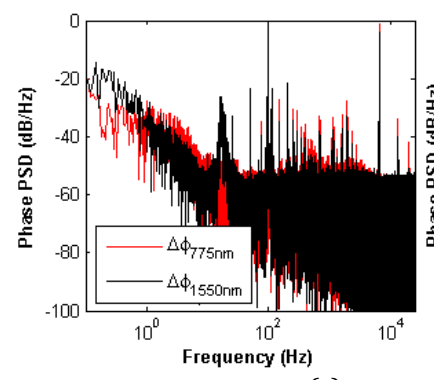

(a)

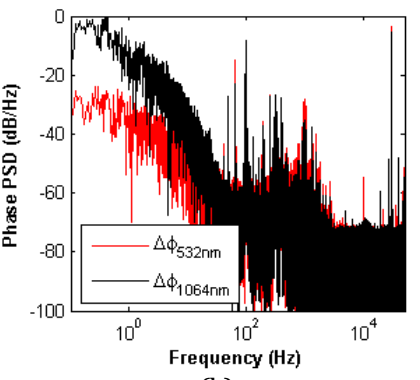

(b)
Fig. 4. Phase spectral density of the harmonic and fundamental waves when the phase control loop operates on the interference signal between the harmonic beams: (a) for the PPLN experiment (b) for the LBO experiment.

Figure 4a reveals that most of the residual phase fluctuations of the fundamental waves are at very low frequency $(\leq 5 \mathrm{~Hz})$, which is typical of the slow variations induced by thermal effects. Peaks at $50 \mathrm{~Hz}$ and $100 \mathrm{~Hz}$ are visible on the PSD curve corresponding to residual electrical noise.

Figure $4 \mathrm{~b}$ presents the PSD measurements from the LBO experiment. Similar conclusions can be made: phase control operating on the second harmonic wave's interference signal results in efficient combining with strong damping of the harmonic residual phase difference fluctuations. Low frequency $(<50 \mathrm{~Hz})$ phase difference fluctuations remain on the fundamental waves, most probably related to slow optical path length variations induced by temperature changes or atmospheric turbulence perturbations of the laser beams.

The peak at $96 \mathrm{~Hz}$ on the PSD graph of the LBO experiment Fig $4 \mathrm{~b}$ corresponds to a strong sinusoidal modulation of the phase difference between the fundamental. As this parasitic phase difference modulation is far below the $4-\mathrm{kHz}$ cut-off frequency limit of the feedback loop, it was easily compensated for in the combining process at $2 \omega$. However, it prevented simultaneous combining of the fundamental waves, as the interference signal at $\omega$ was still modulated at $96 \mathrm{~Hz}$. The source of this modulation has been thoroughly investigated but remains unknown.

In this paper, two experimental demonstrations of coherent combining of second harmonic converters by active phase control using all-fiber components are presented and investigated.

Direct measurements of the residual phase difference between the fundamental beams and between the harmonic beams experimentally confirm the fixed relation existing between the fundamental and the second harmonic phases.

The feasibility of nonlinear frequency converter combining through indirect control of the converted-beams phases is experimentally proven.

Efficient combining of pairs of second harmonic generators is achieved for two different fundamental wavelengths: $1.55 \mu \mathrm{m}$ and $1.06 \mu \mathrm{m}$. The residual phase error is lower than $\lambda / 30 \mathrm{rms}$ in both these SHG CBC experiments.

These experimental tests reveal that simultaneous combining of both fundamental waves and second harmonic waves can be achieved when thermal and turbulence effects do not induce significant optical path length fluctuations on the fundamental beams. However, in this case, as the phase control process operates on the interference signal between the harmonic waves, the fundamental waves are combined with a lower efficiency.

Obviously, the phase relation also exists for three wave nonlinear processes like parametric amplification. Thus, these techniques of indirect phase control can be expanded to other nonlinear devices, such as difference frequency generators, optical parametric amplifiers or optical parametric oscillators. Future work will be dedicated to coherent mid-infrared beam combining by use of DFG and $\mathrm{OPO}$ converters pumped by $1-\mu \mathrm{m}$ fiber lasers.

\section{Funding}

This work was partially funded by Delegation Generale pour l'Armement and Region Ile-de-France.

\section{References}

1. G. D. Goodno, H. Komine, S. J. McNaught, S. B. Weiss, S. Redmond, W. Long, R. Simpson, E. C. Cheung, D. Howland, P. Epp, M. Weber, M. McClellan, J. Sollee and H. Injeyan, Opt. Lett. 31 (9), 1247-1249 (2006)

2. S. J. McNaught, H. Komine, S. B. Weiss, R. Simpson, A. M. F. Johnson, J. Machan, C. P. Asman, M. Weber, G. C. Jones, M. M. Valley, A. Jankevics, D. Burchman, M. McClellan, J. Sollee, J. Marmo and H. Injeyan, in OSA Technical Digest Series (2009), paper CThA1

3. J. Bourderionnet, C. Bellanger, J. Primot and A. Brignon, Opt. Express 19 (18), 17053-17058 (2011)

4. T. M. Shay, J. T. Baker, C. A. Robin, Lt. C. Vergien, C. Zerinque, D. Gallant, A. D. Sanchez, Sgt. D. Pilkington, C. A. Lu and Capt. T. J. Bronder, in LEOS (2008), paper ThL1, pp. 783-784

5. A. Flores, T. M. Shay, C. A. Lu, C. Robin, B. Pulford, A. D Sanchez, D. W Hult and K. B Rowland, in OSA Technical Digest Series (2011), paper CFE3

6. S. M. Redmond, D. J. Ripin, C. X. Yu, S. J. Augst, T. Y. Fan, P. A. Thielen, J. E. Rothenberg and G. D. Goodno, Opt. Lett. 37 (14), 2832-2834 (2012)

7. V. Jolivet, P. Bourdon, B. Bennal, L. Lombard, D. Goular, E. Pourtal, G. Canat, Y. Jaouen, B. Moreau and O. Vasseur, IEEE J. Sel. Top. Quant. Elec. 15 (2), 257-268 (2009)

8. T. Weyrauch, M. A. Vorontsov, G. W. Carhart, L. A. Beresnev, A. P. Rostov, E. E. Polnau and J. Jiang Liu, Opt. Lett. 36 (22), 4455-4457 (2011)

9. B. Pulford, T. Shay, J. Baker, A. Flores, C. Robin, Lt. C. Vergien, C. Zeringue, D. Gallant, A. D. Sanchez, C. Lu and A. Lucero, in OSA Technical Digest Series (2010), paper CThO5

10. A. Liu, M. A. Norsen and R. D. Mead, Opt. Lett. 30 (1), 67-69 (2005)

11. C. D. Brooks and F. Di Teodoro, Opt. Comm. 280 (2), 424-430 (2007)

12. A. V. Smith and D. J. Armstrong, J. Opt. Soc. Am. B 19 (8), 1801-1814 (2002)

13. C. Kieleck, M. Eichhorn, A. Hirth, D. Faye and E. Lallier, Opt. Lett. 34 (3), 262-264 (2009)

14. E. Lippert, G. Rustad and K. Stenersen, Proc. SPIE 6738, 67380D (2007)

15. Y. Q. Zheng, H. Y. Zhu, L. X. Huang, H. B. Chen, Y. M. Duan, R. B. Su, C. H. Huang, Y. Wei, J. Zhuang and G. Zhang, Laser Phys. 20 (4), 756-760 (2010)

16. E. Lippert, H. Fonnum, G. Arisholm and K. Stenersen, Opt. Express 18 (25), 26475-26483 (2010)

17. L. R. Taylor, Y. Feng and D. Bonaccini Calia, Opt. Express 18 (8), 8540-8555 (2010)

18. S.-W. Chiow, T. Kovachy, J. M. Hogan and M. A. Kasevich, Opt. Lett. 37 (18), 3861-3863 (2012)

19. P. Zhang, Y. Guan, X. Xie, J. Zhou, L. Yan and K. S. Wong, Opt. Express 18 (3), 2995-2999 (2010)

20. Y. Guan, P. Zhang, X. Xie, J. Zhou and K. S. Wong, IEEE J. Quant. Elec. 47 (3), 348-353 (2011)

21. P. Bourdon, A. Durécu, G. Canat, J. Le Gouët, D. Goular and L. Lombard, Proc. SPIE 9344, 93441S (2015)

22. Armstrong, J. A., Bloembergen, N., Ducuing, J., \& Pershan, P. S. Physical Review, 127 (6), 1918 (1962). 
Full references (for reviewing needs only)

1. G. D. Goodno, H. Komine, S. J. McNaught, S. B. Weiss, S. Redmond, W. Long, R. Simpson, E. C. Cheung, D. Howland, P. Epp, M. Weber, M. McClellan, J. Sollee and H. Injeyan, "Coherent combination of high-power, zigzag slab lasers, "Opt. Lett. 31 (9), 1247-1249 (2006)

2. S. J. McNaught, H. Komine, S. B. Weiss, R. Simpson, A. M. F. Johnson, J. Machan, C. P. Asman, M. Weber, G. C. Jones, M. M. Valley, A. Jankevics, D. Burchman, M. McClellan, J. Sollee, J. Marmo and H. Injeyan, "100 kW coherently combined slab MOPAs," in Conference on Lasers and Electro-Optics/International Quantum Electronics Conference, OSA Technical Digest Series (Optical Society of America, 2009), paper CThA1

3. J. Bourderionnet, C. Bellanger, J. Primot and A. Brignon, "Collective coherent phase combining of 64 fibers," Opt. Express 19 (18), 17053-17058 (2011)

4. T. M. Shay, J. T. Baker, C. A. Robin, Lt. C. Vergien, C. Zerinque, D. Gallant, A. D. Sanchez, Sgt. D. Pilkington, C. A. Lu and Capt. T. J. Bronder, "Electronic phasing of high power fiber amplifier arrays," in Proceeding of the $21^{\text {st }}$ Annual Meeting of the IEEE Lasers and Electro-Optics Society - LEOS 2008 (Institute of Electrical and Electronics Engineers, 2008), paper ThL1, pp. 783-784

5. A. Flores, T. M. Shay, C. A. Lu, C. Robin, B. Pulford, A. D Sanchez, D. W Hult and K. B Rowland, "Coherent beam combining of fiber amplifiers in a $k W$ regime," in Conference on Lasers and Electro-Optics, OSA Technical Digest Series (Optical Society of America, 2011), paper CFE3

6. S. M. Redmond, D. J. Ripin, C. X. Yu, S. J. Augst, T. Y. Fan, P. A. Thielen, J. E. Rothenberg and G. D. Goodno, "Diffractive coherent combining of a $2.5 \mathrm{~kW}$ fiber laser array into a $1.9 \mathrm{~kW}$ Gaussian beam," Opt. Lett. 37 (14), 2832-2834 (2012)

7. V. Jolivet, P. Bourdon, B. Bennar, L. Lombard, D. Goular, E. Pourtal, G. Canat, Y. Jaouen, B. Moreau and O. Vasseur, "Beam shaping of single-mode and multimode fiber amplifier arrays for propagation through atmospheric turbulence," IEEE J. Sel. Top. Quant. Elec. 15 (2), 257-268 (2009)

8. T. Weyrauch, M. A. Vorontsov, G. W. Carhart, L. A. Beresnev, A. P. Rostov, E. E. Polnau and J. Jiang Liu, "Experimental demonstration of coherent beam combining over a $7 \mathrm{~km}$ propagation path," Opt. Lett. 36 (22), 44554457 (2011)

9. B. Pulford, T. Shay, J. Baker, A. Flores, C. Robin, Lt. C. Vergien, C. Zeringue, D. Gallant, A. D. Sanchez, C. Lu and A. Lucero, "Phase locking an array of fiber amplifiers onto a remote object," in Conference on Lasers and ElectroOptics/Quantum Electronics and Laser Science Conference, OSA Technical Digest Series (Optical Society of America, 2010), paper CThO5

10. A. Liu, M. A. Norsen and R. D. Mead, "60-Wgreen output by frequency doubling of a polarized Yb-doped fiber laser," Opt. Lett. 30 (1), 67-69 (2005)
11. C. D. Brooks and F. Di Teodoro, "High peak power operation and harmonic generation of a singlepolarization, Yb-doped photonic crystal fiber amplifier," Opt. Comm. 280 (2), 424-430 (2007)

12. A. V. Smith and D. J. Armstrong, "Nanosecond optical parametric oscillator with $90^{\circ}$ image rotation: design and performance, "J. Opt. Soc. Am. B 19 (8), 1801-1814 (2002)

13. C. Kieleck, M. Eichhorn, A. Hirth, D. Faye and E. Lallier, "High-efficiency $20-50 \mathrm{kHz}$ mid-infrared orientationpatterned GaAs optical parametric oscillator pumped by a $2 \mu \mathrm{m}$ holmium laser, "Opt. Lett. 34 (3), 262-264 (2009)

14. E. Lippert, G. Rustad and K. Stenersen, "High power and efficient far infrared $\mathrm{ZnGeP}$-based optical parametric oscillator, "Proc. SPIE 6738, 67380D (2007)

15. Y. Q. Zheng, H. Y. Zhu, L. X. Huang, H. B. Chen, Y. M. Duan, R. B. Su, C. H. Huang, Y. Wei, J. Zhuang and G. Zhang, "Efficient 532-nm laser using high gray tracking resistance KTP crystal,"Laser Phys. 20 (4), 756-760 (2010)

16. E. Lippert, H. Fonnum, G. Arisholm and K. Stenersen, "A 22-watt mid-infrared optical parametric oscillator with $V$ shaped 3-mirror ring resonator," Opt. Express 18 (25), 26475-26483 (2010)

17. L. R. Taylor, Y. Feng and D. Bonaccini Calia, "50W CW visible laser source at 589nm obtained via frequency doubling of three coherently combined narrow-band Raman fibre amplifiers," Opt. Express 18 (8), 8540-8555 (2010)

18. S.-W. Chiow, T. Kovachy, J. M. Hogan and M. A. Kasevich, "Generation of $43 \mathrm{~W}$ of quasi-continuous 780 nm laser light via high-efficiency, single-pass frequency doubling in periodically poled lithium niobate crystals," Opt. Lett. 37 (18), 3861-3863 (2012)

19. P. Zhang, Y. Guan, X. Xie, J. Zhou, L. Yan and K. S. Wong, "Phase controlled beam combining with nonlinear frequency conversion," Opt. Express 18 (3), 2995-2999 (2010)

20. Y. Guan, P. Zhang, X. Xie, J. Zhou and K. S. Wong, "Coherent beam combining with second-harmonic generation optimized with adaptive phase control," IEEE J. Quant. Elec. 47 (3), 348-353 (2011)

21. P. Bourdon, A. Durécu, G. Canat, J. Le Gouët, D. Goular and L. Lombard, "Coherent combining of fiber-laserpumped frequency converters using all-fiber electro-optic modulator for active phase control," Proc. SPIE 9344, 93441S (2015)

22. Armstrong, J. A., Bloembergen, N., Ducuing, J., \& Pershan, P. S. "Interactions between light waves in a nonlinear dielectric'. Physical Review, 127 (6), 1918 (1962). 